\title{
Mechanistic Studies of Cytochrome P450 3A4 Time-Dependent Inhibition Using Two Cysteine-Targeting Electrophiles ${ }^{\text {\} }$
}

\author{
John T. Barr, Zhican Wang, Xiaoshan Min, Henry J. Wienkers, Brooke M. Rock, Dan A. Rock, \\ and Larry C. Wienkers
}

Amgen Research, Pharmacokinetics and Drug Metabolism, Amgen Inc., South San Francisco, California

Received October 24, 2019; accepted February 21, 2020

\section{ABSTRACT}

Experiments designed to identify the mechanism of cytochrome P450 inactivation are critical to drug discovery. Small molecules irreversibly inhibit P450 enzymatic activity via two primary mechanisms: apoprotein adduct formation or heme modification. Understanding the interplay between chemical structures of reactive electrophiles and the impact on CYP3A4 structure and function can ultimately provide insights into drug design to minimize P450 inactivation. In a previous study, raloxifene and N-(1-pyrene) iodoacetamide (PIA) alkylated CYP3A4 in vitro; however, only raloxifene influenced enzyme activity. Here, two alkylating agents with cysteine selectivity, PIA and pyrene maleimide (PM), were used to investigate this apparent compound-dependent disconnect between CYP3A4 protein alkylation and activity loss. The compound's effect on 1) enzymatic activity, 2) carbon monoxide (CO) binding capacity, 3) intact heme content, and 4) protein conformation were measured. Results showed that PM had a large time-dependent loss of enzyme activity, whereas PIA did not. The differential effect on enzymatic activity between PM and PIA was mirrored in the $\mathrm{CO}$ binding data. Despite disruption of $\mathrm{CO}$ binding, neither compound affected the heme concentrations, inferring there was no destruction or alkylation of the heme. Lastly, differential scanning fluorescence showed PM-treated CYP3A4 caused a shift in the onset temperature required to induce protein aggregation, which was not observed for CYP3A4 treated with PIA. In conclusion, alkylation of CYP3A4 apoprotein can have a variable impact on catalytic activity, $\mathrm{CO}$ binding, and protein conformation that may be compounddependent. These results highlight the need for careful interpretation of experimental results aimed at characterizing the nature of P450 enzyme inactivation.

\section{SIGNIFICANCE STATEMENT}

Understanding the mechanism of CYP3A4 time-dependent inhibition is critical to drug discovery. In this study, we use two cysteinetargeting electrophiles to probe how subtle variation in inhibitor structure may impact the mechanism of CYP3A4 time-dependent inhibition and confound interpretation of traditional diagnostic experiments. Ultimately, this simplified system was used to reveal insights into CYP3A4 biochemical behavior. The insights may have implications that aid in understanding the susceptibility of CYP enzymes to the effects of electrophilic intermediates generated via bioactivation.

\section{Introduction}

Experiments aimed at identifying the mechanistic basis behind cytochrome P450 (P450) inactivation represent a critical line of investigation during drug discovery. Early understanding of enzyme inactivation can inform rational chemical modifications within a chemical series, ultimately mitigating the mechanism-based inactivation (MBI) liability. In cases in which P450 MBI cannot be avoided or is overlooked, the clinical importance can be profound. Inactivation of P450 enzymes can lead to serious drug-drug interactions and, in some instances, removal of the drug from the market (Orr et al., 2012). Concerns for MBI liability are particularly important for CYP3A4, as this isozyme is responsible for the metabolism of approximately $50 \%$ of current marketed drugs (Zhou, 2008). To this end, the application of enzyme kinetic studies and mechanistic experiments aimed to understand the chemical mechanism for CYP3A4 MBI remains critical. Tools to investigate MBI mechanism need to be rapid to enable

https://doi.org/10.1124/dmd.119.089813.

S This article has supplemental material available at dmd.aspetjournals.org. structure-activity-based design in line with the speed of chemical synthesis.

P450 MBI by definition results in reduced P450 enzymatic activity and can be achieved via two primary mechanisms: apoprotein adduction or heme modification (Zhou et al., 2007; Foti et al., 2011). Examples of P450 inactivation through either mechanism are numerous. For example, raloxifene, dihydroxybergamottin, and ritonavir have been shown to covalently bind to P450 apoprotein, leading to potent enzymatic inactivation (Bateman et al., 2004; Baer et al., 2007; Rock et al., 2014). In contrast, 1-aminobenzotriazole (ABT), mibefradil, and fluoxetine elicit P450 inactivation by modifying the heme moiety via heme alkylation, heme destruction, or coordinated bond formation with the heme iron, respectively (Murray and Murray, 2003; VandenBrink and Isoherranen, 2010; Foti et al., 2011; de Montellano, 2018). The mechanism of inactivation is intimately linked to the chemistry involved in the bioactivation and subsequent reactive species associated with each compound.

A variety of in vitro diagnostic methods such as whole protein mass spectrometry, UV-visible spectroscopy, and kinetic analysis have been used to generate experimental observations to characterize the nature of

ABBREVIATIONS: ABT, 1-aminobenzotriazole; CO, carbon monoxide; CYP3A4, cytochrome P450 3A4; CYP3A5, cytochrome P450 3A5; DSF, differential scanning fluorescence; GSH, glutathione; LC, liquid chromatography; MBI, mechanism-based inactivation; MS/MS, tandem mass spectrometry; P450, cytochrome P450; PIA, N-(1-pyrene)iodoacetamide; PM, pyrene maleimide $\mathrm{T}_{\text {agg }}$ aggregation temperature. 
the MBI. Experimental observations can often be complex, and in some cases, evidence may point to multiple mechanisms simultaneously occurring from the same substrate (Lin et al., 2002, 2018). As a further indication of complexity, it has been previously shown that covalent enzyme modification does not necessarily cause loss of function, depending on the location of the adduct and likely on chemical structure of the compound (Baer et al., 2007).

In previous work, it was demonstrated that CYP3A4 covalent modification by a small molecule did not necessarily precipitate a loss of enzyme function; raloxifene and PIA both alkylate Cysteine 239 of CYP3A4 in vitro; however, only raloxifene produced a measurable inactivation rate (Baer et al., 2007). Here, we use two electrophilic alkylating agents to mechanistically investigate this apparent compounddependent disconnect between covalent protein modification and activity loss. These tool compounds offer the advantage of containing a highly reactive electrophilic group allowing for facile alkylation of CYP3A4 protein without requiring enzyme-catalyzed bioactivation (Fig. 1). Furthermore, the pyreneyl moiety was chosen to enhance the affinity of the molecules toward the CYP3A4 enzyme active site (Dabrowski et al., 2002; Jushchyshyn et al., 2005). The direct alkylation provides a controlled system to evaluate the experimental tools used to study P450 MBI more thoroughly, including: 1) P450 catalytic activity, 2) carbon monoxide heme binding capacity, 3) heme quantitation, 4) P450 alkylation, and 5) thermodynamic protein conformation analysis. Using this simplified system to further understand the precise mechanistic features of CYP3A4 inactivation may serve as a tool to ultimately reveal insights into fundamental CYP3A4 biochemistry and the enzyme's susceptibility to the effects of electrophilic intermediates generated via bioactivation.

\section{Materials and Methods}

Materials. $N$-(1-pyrenyl)maleimide, $N$-(1-pyrenyl)iodoacetamide, NADPH, glutathione (GSH), ABT, meso-tetra(4-carboxyphenyl)porphine, midazolam, tolbutamide, and raloxifene were purchased from Sigma Aldrich (St. Louis, MO). Supersomes containing recombinantly expressed CYP3A4 and CYP3A5 derived from baculovirus-transfected insect cells were purchased from Corning (NY). Purified recombinant CYP3A4 was received as a gift from Prof. Jeffrey Jones; the protein was expressed and purified as described previously (Barnaba et al., 2016). All other chemicals and reagents were purchased from Sigma Aldrich or Fisher Scientific (Pittsburgh, PA) unless stated otherwise.

Time-Dependent Inactivation of CYP3A4 and CYP3A5 Activity. Both PIA and PM were tested for time-dependent inhibition in CYP3A4 and CYP3A5 Supersomes containing NADPH reductase. In brief, $50 \mu \mathrm{M}$ of each test compound (or DMSO as vehicle control) was prewarmed for 5 minutes in $100 \mathrm{mM}$ potassium phosphate buffer ( $\mathrm{pH} 7.4$ ) containing $3 \mathrm{mM} \mathrm{MgCl}_{2}$. Reactions were initiated by the addition of CYP3A Supersomes (final concentration of $50 \mathrm{pmol} / \mathrm{ml} \mathrm{CYP} 3 \mathrm{~A}$ ) and proceeded for multiple time points up to 30 minutes at $37^{\circ} \mathrm{C}$. After the preincubation period, a 20 -fold dilution was made into a secondary assay containing $10 \mu \mathrm{M}$ midazolam, $3 \mathrm{mM} \mathrm{MgCl} 2,1 \mathrm{mM} \mathrm{NADPH}$, and $100 \mathrm{mM}$ potassium phosphate buffer ( $\mathrm{pH}$ 7.4). Secondary incubations were quenched after
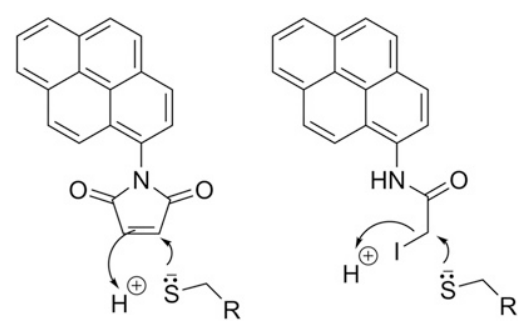

Fig. 1. Structures of PM and PIA. PM (left) and PIA (right) differ by the electrophilic group attached to a pyrene core. Arrows indicate the flow of electrons from an attacking cysteine thiolate anion nucleophile.
5 minutes at $37^{\circ} \mathrm{C}$ with three volumes of acetonitrile containing $1 \mu \mathrm{g} / \mathrm{ml}$ tolbutamide as an internal standard. Enzyme activity was measured by monitoring the formation of 1-OH-midazolam using liquid chromatography (LC)-tandem mass spectrometry (MS/MS) as previously described (Foti et al., 2011).

Mass Spectral Analysis of Intact CYP3A4 Protein. PIA or PM $(5 \mu \mathrm{M})$ were separately incubated with purified CYP3A4 $(0.5 \mu \mathrm{M})$ in $0.1 \mathrm{M}$ potassium phosphate $(\mathrm{pH} 7.4)$ buffer in a total volume of $0.2 \mathrm{ml}$. The incubation was conducted for 10 minutes, at which point a $100-\mu l$ aliquot was transferred to a Microcon centrifugal filter tube (30-kDa filter) and centrifuged for 5 minutes at $10,000 \mathrm{~g}$. The sample was analyzed on an LTQ Orbitrap Velos mass spectrometer (Thermo Fisher Scientific, San Jose, CA) coupled to an Accela 1250 HPLC system with a Leap CTC PAL autosampler (Leap Technologies, Carrboro, NC). Initial HPLC conditions were $82 \%$ solvent $\mathrm{A}\left(0.05 \%\right.$ trifluoroacetic acid in $\left.\mathrm{H}_{2} \mathrm{O}\right)$ and $18 \%$ solvent $\mathrm{B}(0.05 \%$ trifluoroacetic acid in acetonitrile) at a flow rate of $250 \mu \mathrm{l} / \mathrm{min}$ The following linear gradient elution profile was used: $18 \% \mathrm{~B}$ for 1 minute, $18 \%-90 \%$ solvent B for 12 minutes, and $90 \%$ solvent B for 2 minutes separated on a Biobasics 4 column (Thermo Fisher Scientific). LTQ Orbitrap parameters were set as follows: source temperature, $350^{\circ} \mathrm{C}$; sheath gas, 35 arbitrary units; auxiliary gas, 5 arbitrary units; spray voltage, $3 \mathrm{kV}$; capillary temperature, $300^{\circ} \mathrm{C}$; S-lens radiofrequency, $85 \%$; resolution, 15,000; ion trap and Orbitrap maximum injection time, 500 milliseconds; and $\mathrm{m} / \mathrm{z}$ scan range, 650-1850 Da. Mass spectra were averaged over the entire width of the peak and deconvoluted using ProMass 2.0 software (Novatia, Monmouth Junction, NJ).

Time-Dependent Measurement of Carbon Monoxide Binding Loss. Absorbance measurements were taken from a range of 400-500 nm using a Cary 4000 UV-visible dual beam spectrometer (Varian Inc., Palo Alto, CA). Sample cuvettes contained 500 pmol CYP3A4 with $\mathrm{P} 450$ reductase and either PM $(50 \mu \mathrm{M})$ or PIA $(50 \mu \mathrm{M})$ in $0.1 \mathrm{M}$ potassium phosphate buffer (pH 7.4) containing $10 \%$ glycerol. Reactions were initiated by the addition of PM or PIA after prewarming mixtures at $37^{\circ} \mathrm{C}$ for 5 minutes. After incubations proceeded for certain time points as indicated, the sample cuvettes were bubbled with $\mathrm{CO}$ gas for 1 minute (one bubble per second), and approximately $1 \mathrm{mg}$ sodium dithionite was added to both the sample and reference cuvettes (Rock et al., 2014). The reduced CO difference spectra were recorded by scanning from 400 to $500 \mathrm{~nm}$ after normalizing to the baseline. To minimize the background noise from PM or PIA, the reference cuvette contained identical components as sample cuvette except for CO (i.e., CYP3A4, buffer, glycerol, test article, and sodium dithionite). Spectra from different incubation time points were normalized to one another based on absorbance at $475 \mathrm{~nm}$.

In addition, raloxifene and ABT were used as negative and positive control for $\mathrm{CO}$ binding loss, respectively. Incubation mixtures contained $500 \mathrm{pmol}$ of CYP3A4 with P450 reductase, $1 \mathrm{mM}$ NADPH, and either vehicle $(0.1 \%$ DMSO $\mathrm{v} / \mathrm{v})$, raloxifene $(50 \mu \mathrm{M})$, or ABT $(500 \mu \mathrm{M})$ in $0.1 \mathrm{M}$ potassium phosphate buffer ( $\mathrm{pH} 7.4)$. Reactions were initiated by the addition of NADPH and conducted up to 30 minutes at $37^{\circ} \mathrm{C}$. The reduced $\mathrm{CO}$ spectra were recorded as described above after normalizing to a baseline generated by reference cuvettes containing identical components except for CO. Spectra from different incubation time points were normalized to one another based on absorbance at $475 \mathrm{~nm}$.

LC-UV-MS/MS Analysis of Total Intact Heme Loss. After incubation of CYP3A4 (40 pmol/ml, with NADPH-P450 reductase and without b5) in phosphate buffer (0.1 M, pH 7.4) with $50 \mu \mathrm{M}$ PM or PIA for $0,1,2,5,10$, and 20 minutes at $37^{\circ} \mathrm{C}$, reactions were quenched with 0.1 volume of $1 \mathrm{~N} \mathrm{HCl}$ and one volume acetonitrile ( $3 \%$ formic acid) containing $0.2 \mu \mathrm{M}$ internal standard mesotetra(4-carboxyphenyl)porphine. The percentage of organic solvent (DMSO) in the reaction was $0.5 \%$. The mixed solutions were kept on ice for 5 minutes, followed by centrifugation at $12,000 \mathrm{~g}$ to collect supernatants for LC-UV-MS/MS analysis. Inactivation of CYP3A4 $(40 \mathrm{pmol} / \mathrm{ml})$ by ABT $(200 \mu \mathrm{M})$ and raloxifene $(100 \mu \mathrm{M})$ were conducted in the presence of $1 \mathrm{mM} \mathrm{NADPH}$ at $37^{\circ} \mathrm{C}$. Reactions were quenched with $1 \mathrm{~N} \mathrm{HCl}$ and acetonitrile containing internal standard as described above after incubation for $0,1,2,5,10$, and 20 minutes, followed by supernatant collection. The total reaction volume was $100 \mu$ l. Determination of intact heme was performed on a Thermo Q Exactive orbitrap high-resolution MS coupled with an Accela LC system and a photodiode array detector. Chromatographic separation was achieved on a Thermo Hypersil Gold column $(1.9 \mu \mathrm{m}, 50 \times 2.1 \mathrm{~mm}$ ), with a gradient as follows: $0-0.2$ minutes, $5 \% \mathrm{~B}$; 2.5-3.5 minutes, $95 \% \mathrm{~B}$; and 3.6-5 minutes, $5 \% \mathrm{~B}$ (A: water with $0.1 \%$ formic acid; $\mathrm{B}$ : acetonitrile with $0.1 \%$ formic acid). The flow rate was $0.6 \mathrm{ml} / \mathrm{min}$. A full 
accurate MS scan was performed from $\mathrm{m} / z, 100$ to 1000 with a resolution at 70,000 , followed by a data-dependent acquisition for top six most intense ions at a resolution of 17,500. Intact heme and internal standard were monitored by UV absorbance at $398 \mathrm{~nm}$ as well as accurate mass at $\mathrm{m} / \mathrm{z} 616.1745$ and $791.2106 \pm$ $10 \mathrm{ppm}$, respectively. Integration of peak area was accomplished using the Thermo Xcalibur Quan software, and each peak area ratio of intact heme divided by internal standard was calculated. The percentage of heme remaining was calculated with the following formula: heme remaining $(\%)=$ (ratios of treated samples/ratios of 0-minute control) $\times 100 \%$. Data were obtained in triplicate and plotted using Graphpad Prism version 7.

Extraction of Free Heme Released from the CYP3A4. The total and free intact heme amounts in the $\mathrm{P} 450$ reaction solutions were extracted with $80 \%(\mathrm{v} / \mathrm{v})$ acidic acetone (20\% $1 \mathrm{~N} \mathrm{HCl}$ solution) and neutral acetone ( $20 \%$ water) solutions, respectively. Heme extraction was performed based on previously published methods (Stillman and Gassman, 1978; Espinas et al., 2012). Briefly, noncovalently bound heme is expected to be effectively extracted from the protein precipitates into $80 \%$ acidic acetone. In contrast, neutral acetone only can extract free heme that loosely bound to the surface of protein (Espinas et al., 2012). In the present study, CYP3A4 Supersomes (40 pmol/ml without b5) in $0.1 \mathrm{mM}$ phosphate buffer $(\mathrm{pH} 7.4)$ were incubated with DMSO $(0.5 \%, \mathrm{v} / \mathrm{v}), \mathrm{PM}$ $(50 \mu \mathrm{M})$, or PIA $(50 \mu \mathrm{M})$ for $0.5,3,7$, and 10 minutes at a $37^{\circ} \mathrm{C}$ water bath. At each time point, the reaction mixtures were extracted by either acidic or neutral acetone solution containing $0.2 \mu \mathrm{M}$ meso-tetra(4-carboxyphenyl)porphine as the internal standard, respectively. The solutions were thoroughly mixed and centrifuged at $12,000 \mathrm{~g}$ for 5 minutes, and the supernatants were collected for LC-MS analysis. To determine the acetone extraction efficiency, a two-step extraction procedure was conducted. During the first step, the CYP3A4 solutions were extracted by either acidic or neutral acetone. After removal of supernatants, the protein precipitates were extracted by acidic acetone during the second step. Presumably, acidic acetone extraction during the second step will extract the heme residue from protein pellets.

Protein Stability Measurement. Stability of either CYP3A4 alone or treated with PIA or PM was determined using the Optim 2 instrument (Unchained Laboratories, Pleasanton, CA). Briefly, the purified recombinant CYP3A4 protein $(0.5 \mathrm{mg} / \mathrm{ml})$ in Tris- $\mathrm{HCl}$ storage buffer $(20 \%$ glycerol $)$ was incubated with either PM, PIA, or pyrene $(50 \mu \mathrm{M})$ at $37^{\circ} \mathrm{C}$ for 10 minutes. The total incubation volume was $50 \mu \mathrm{l}$. The reactions were terminated by the addition of $5 \mu \mathrm{l}$ of $5 \mathrm{mM} \mathrm{GSH}$ ( $0.5 \mathrm{mM}$ final GSH), and the solutions were kept on ice until analysis. After a brief centrifugation step, supernatant aliquots $(9 \mu \mathrm{l})$ were transferred to the 16-well sample cuvettes and heated in $0.3^{\circ} \mathrm{C}$ increments from 20 to $95^{\circ} \mathrm{C}$. The heating rate between temperature intervals was set to $1^{\circ} \mathrm{C} / \mathrm{min}$. All measurements were made in duplicate. Using an excitation wavelength of $266 \mathrm{~nm}$, fluorescence intensity between 300 and $400 \mathrm{~nm}$ was measured and analyzed as function of the temperature using the manufacturer's software. Similarly, static light scattering at $473 \mathrm{~nm}$ as a function of the temperature ramp was measured to determine the onset of aggregation.

\section{Results}

PM but Not PIA Causes a Rapid Loss of CYP3A4 Activity. Inactivation of CYP3A4 by preincubation with either PM or PIA $(50 \mu \mathrm{M})$ was determined by monitoring the formation rate of 1-hydroxymidazolam. As shown in Fig. 2, PM caused a greater than $95 \%$ decrease of CYP3A4 activity over a course of 30 minutes. In contrast, the PIAtreated sample exhibited less than a $30 \%$ decrease in CYP3A4 activity after 30 minutes (Fig. 2).

Both PM and PIA Alkylate CYP3A4. To investigate the potential of PM and PIA to alkylate CYP3A4, intact MS was performed on CYP3A4 after incubations. The deconvoluted MS spectra showed that both PM and PIA alkylated CYP3A4 apo-protein; however, the extent of alkylation varied (Fig. 3). In the control, unmodified native CYP3A4 had a deconvoluted peak corresponding to a molecular weight of 57,275 Da. After incubating with PM, there were two peaks with an observable mass shift (Fig. 3, top panel), which corresponded to two different alkylated forms of CYP3A4, each modified by 6 and 7 PM molecules, respectively. The predicted theoretical mass shift

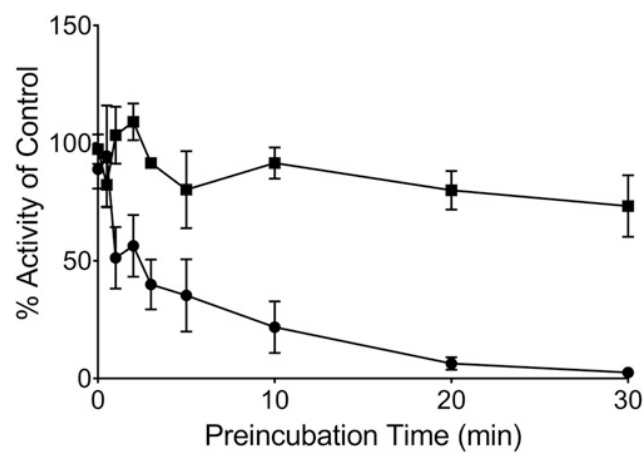

Fig. 2. Inactivation of $\mathrm{CYP} 3 \mathrm{~A} 4$ by $\mathrm{PM}$ and PIA. A solution containing CYP3A4 Supersomes in phosphate buffer was preincubated with PM or PIA $(50 \mu \mathrm{M})$ for various times. After preincubation, mixture solutions were 20 -fold diluted into a secondary incubation containing $10 \mu \mathrm{M}$ midazolam. 1-hydroxymidazolam formation was monitored, and the activity was calculated as a percentage of the product formation observed at the 0 -minute preincubation time point. The mean of transformed data for relative reaction rate of triplicate determinations \pm S.E. is shown. Circles and squares denote treatment by PM and PIA, respectively.

for binding of a single PM is 297. Incubation of CYP3A4 with PIA resulted in a single peak, and the mass shift indicated the bonding of a single PIA molecule to the protein. The predicted theoretical mass shift for binding of a single PIA is 257 . The incubation with PM or PIA reacted to completion, as no observable unmodified CYP3A4 could be detected.

PM and PIA Have Distinctly Different Effects on CO Binding of CYP3A4. To investigate possible mechanistic differences in CYP3A4 inactivation between PM and PIA, UV-visible spectroscopy of CO binding was performed using CYP3A4 Supersomes. Raloxifene $(50 \mu \mathrm{M})$ and ABT $(500 \mu \mathrm{M})$ were used as negative and positive controls, respectively, for modulation of CO binding (Foti et al., 2011). Representative spectra from CYP3A4 incubations with either PM, PIA, ABT, or raloxifene at wavelengths $400-500 \mathrm{~nm}$ are shown in Fig. 4. Upon incubating the protein with PM $(50 \mu \mathrm{M})$, a rapid (within 0.5 minutes), time-dependent loss of $\mathrm{CO}$ binding capacity was observed, as indicated by a marked absorbance decrease at $450 \mathrm{~nm}$. Absorbance at $422 \mathrm{~nm}$ increased over time (Fig. 4A), which indicates separation of the iron-thiolate bond linking the prosthetic heme group to the CYP3A4 apoprotein. In contrast, incubation with PIA $(50 \mu \mathrm{M})$ for a longer incubation time (up to 20 minutes) caused a negligible (within $20 \%$ of 0 minutes) absorbance decrease at $450 \mathrm{~nm}$, along with a mild increase at $422 \mathrm{~nm}$ (Fig. 4B). As expected, incubation with ABT $(500 \mu \mathrm{M})$ in the presence of NADPH caused loss of CO binding, consistent with known mechanism of covalent modification of the heme. An increase in absorbance at $422 \mathrm{~nm}$ was not observed, which was unique compared with that in PM- or PIA-treated samples. Raloxifene, which inactivates CYP3A4 through alkylation of the apoprotein, did not precipitate a loss of $\mathrm{CO}$ binding after a 30-minute incubation in the presence of NADPH (Fig. 4C).

Neither PM nor PIA Precipitate Observable Loss of Total Intact Heme. Intact heme was extracted from incubation mixtures under acidic conditions as described in Materials and Methods. Time-dependent intact heme loss was monitored after treatment of CYP3A4 with vehicle, PM, or PIA $(50 \mu \mathrm{M})$ up to 20 minutes. As shown in Fig. 5, no difference in intact heme was observed for either PM- or PIA-treated samples compared with the vehicle-treated group (heme remaining was $>80 \%$ after 20 minutes). ABT $(500 \mu \mathrm{M})$, known as a covalent modulator of heme, caused a marked loss of intact heme over incubation time (Fig. 5). Raloxifene inactivates CYP3A4 through cysteine modification (Baer et al., 2007; VandenBrink et al., 2012); as 

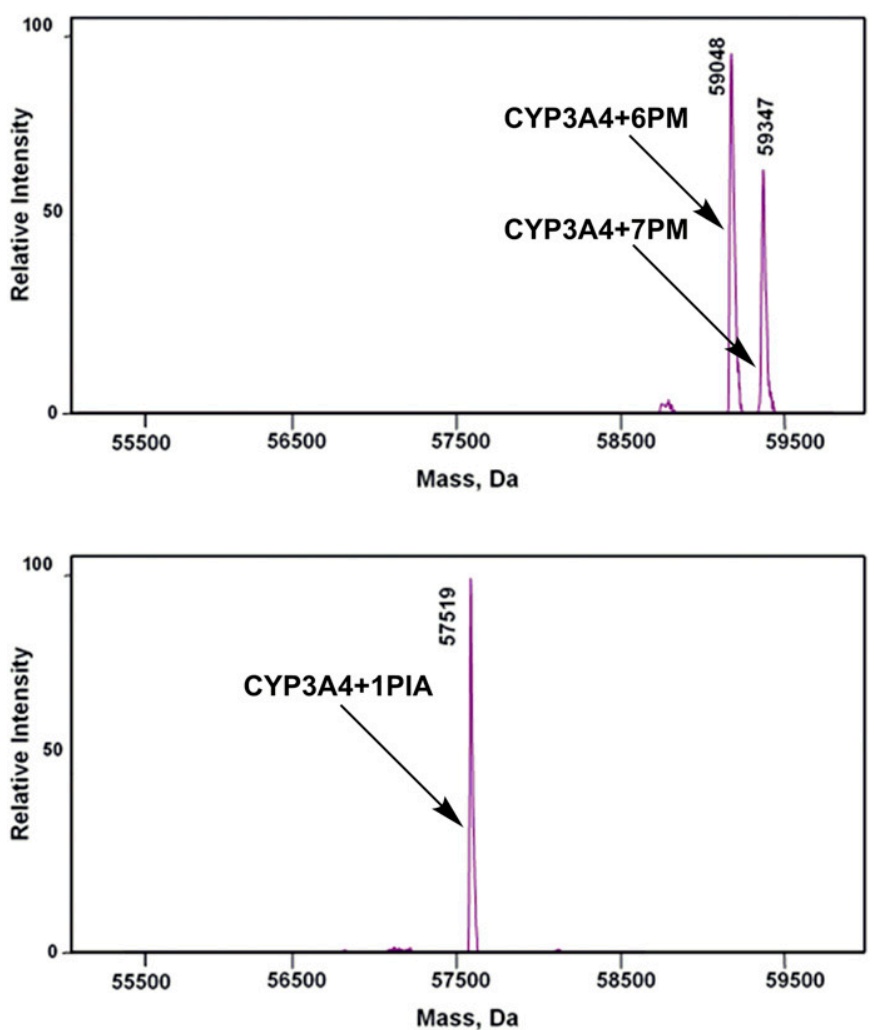

Fig. 3. Alkylation of CYP3A4 apo-protein by PM and PIA. Purified recombinant CYP3A4 $(0.5 \mu \mathrm{M})$ was reacted with of PIA or PM $(5 \mu \mathrm{M})$ in separate tubes for 10 minutes. The samples were analyzed by high-resolution mass spectrometry, and resultant deconvoluted spectra are shown in the chromatogram for PM (top panel) and PIA (bottom panel).

expected, preincubation of CYP3A4 with raloxifene resulted in a negligible loss of intact heme.

PM Treatment Precipitates Release of Free Heme from CYP3A4. Although both PM and PIA are capable of alkylating CYP3A4, only PM caused major CO binding loss of CYP3A4, which is unlikely related to covalent modification of heme. To measure any differences in the amount of free heme (i.e., heme in solution that is not covalently bound to P450), we compared the difference of free heme from CYP3A4 after treatment by PM and PIA. As shown in Fig. 6, PM induced a $>50 \%$ increase of free heme released from CYP3A 4 within 3 minutes, whereas PIA only increased free heme release by approximately $20 \%$. After treatment by PIA or PM, total heme was unchanged over time (Fig. 6), which is consistent with previous observations (Fig. 5).

PM Treatment Induces CYP3A4 Protein Instability. Melting temperatures and onset of aggregation temperatures were determined using an Optim 2 differential scanning fluorescence (DSF) system, which combines intrinsic fluorescence and static light scattering measurements using the same low-volume sample in high-throughput mode. Intrinsic tryptophan spectrum shifts to red wavelengths because of a hydrophobic to hydrophilic change in chemical environment. The simultaneously measured static light scattering evaluates the aggregation formation within the applied thermal shift (Temel et al., 2016). Because the compounds tested (PM, PIA, or pyrene) interfere with the intrinsic tryptophan fluorescence, we were not able to obtain accurate measurement of the melting temperature in the presence of compounds. However, the aggregation temperature $\left(\mathrm{T}_{\mathrm{agg}}\right)$ was readily measured, and this value can serve as an accurate parameter to measure the inherent colloidal stability of protein. In the absence of compound, CYP3A4 has an onset $\mathrm{T}_{\text {agg }}$ value at $47.7^{\circ} \mathrm{C}$. As shown in Table 1 , treatment with either PIA or pyrene did not have a meaningful effect on the onset of $\mathrm{T}_{\mathrm{agg}}$. However, treatment of CYP3A4 with PM decreased onset $\mathrm{T}_{\mathrm{agg}}$ by more than $10^{\circ}$, with a value of $36^{\circ} \mathrm{C}$. Representative aggregation curves for treatments of recombinant CYP3A4 are shown in Supplemental Fig. 1.

Treatment of CYP3A5 with either PM or PIA Does Not Alter Enzymatic Activity or CO Binding. A solution of CYP3A5 Supersomes was treated with either PM $(50 \mu \mathrm{M})$, PIA $(50 \mu \mathrm{M})$, or vehicle control, and enzymatic activity was monitored by following 1hydroxymidazolam formation. Over the course of 30 minutes, the enzyme activity remained within $20 \%$ of vehicle control, indicating neither PM nor PIA had a time-dependent effect on activity (Fig. 7A). Similarly, PM or PIA treatment of CYP3A5 did not alter the CO binding capacity of the enzyme after incubation by more than $20 \%$ relative to vehicle control (Fig. 7B).

\section{Discussion}

The study of P450 MBI is an important component of drug discovery. Timely recognition of P450 MBI liability can have profound implications on patient safety and clinical outcomes. If MBI is identified early in

A

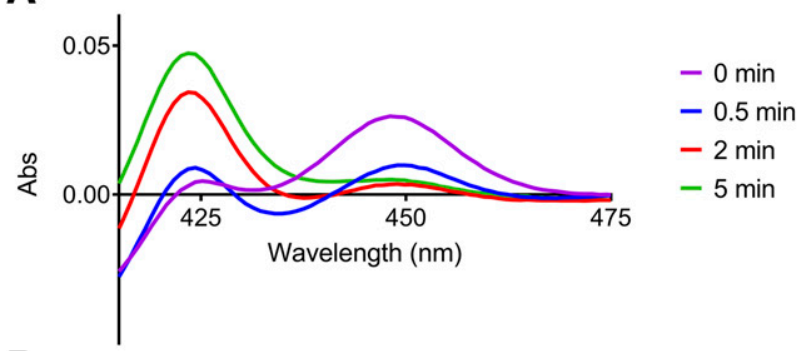

B

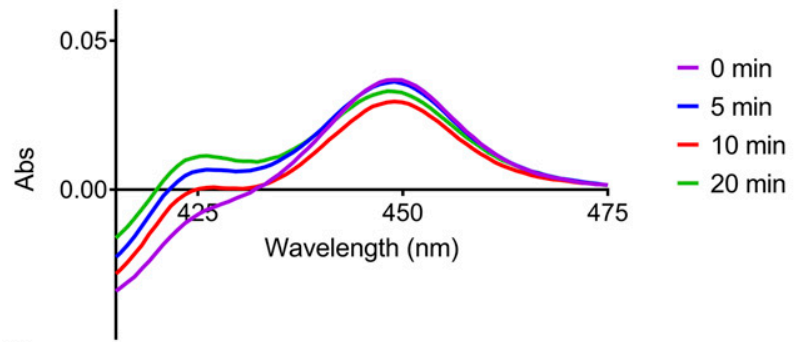

C

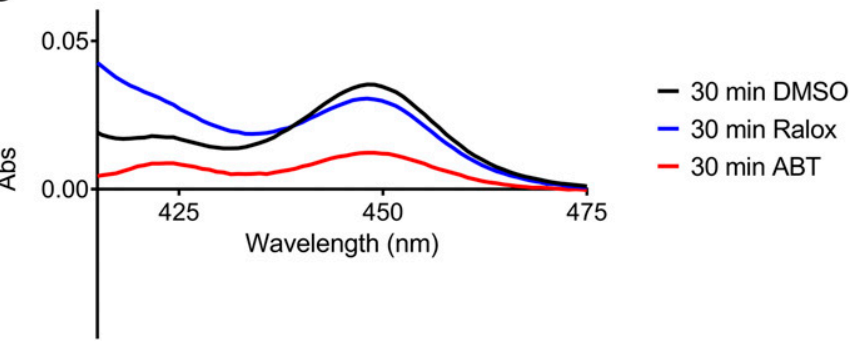

Fig. 4. Effect of small molecule treatment on CYP3A4 CO binding capacity. (A) Incubations containing CYP3A4 Supersomes were treated with PM $(50 \mu \mathrm{M})$. After incubation of CYP3A4 with PM for $0,0.5,2$, and 5 minutes at $37^{\circ} \mathrm{C}$, CO binding was measured using UV-visible difference spectra as described in Materials and Methods. (B) Incubation CYP3A4 Supersomes with PIA $(50 \mu \mathrm{M})$. After incubation of CYP3A4 with PM for $0,5,10$, and 20 minutes, $\mathrm{CO}$ binding was measured using UV-visible difference spectra as described in Materials and Methods. (C) Incubation CYP3A4 Supersomes with either ABT or raloxifene in the presence of NADPH. Reaction was initiated by the addition of NADPH and incubated for 30 minutes at $37^{\circ} \mathrm{C}$. 


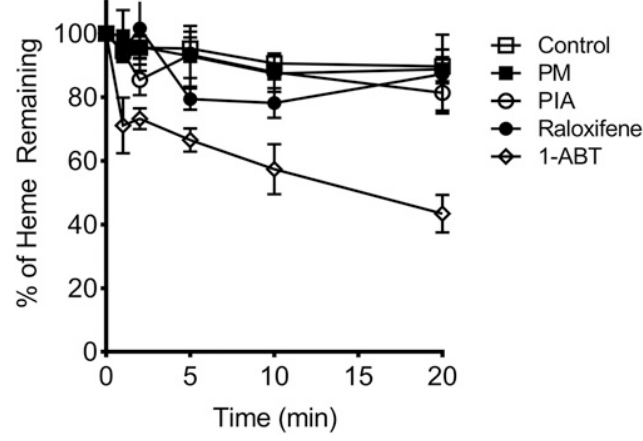

Fig. 5. Quantitative assessment of intact heme loss after CYP3A4 treatment with PM and PIA. CYP3A4 Supersomes (4 pmol) were incubated with vehicle (0.5\% DMSO), PM $(50 \mu \mathrm{M})$, PIA $(50 \mu \mathrm{M})$, ABT $(200 \mu \mathrm{M})$, and raloxifene $(100$ $\mu \mathrm{M})$ as described in the Materials and Methods section. After an incubation of 0,1 , $2,5,10$, and 20 minutes, aliquots were quenched and extracted for LC-UV-MS/MS analysis. The percentage of intact heme remaining after treatment is shown. Points and error bars represent the mean of triplicate experiments \pm S.E.

lead optimization, mitigation of the bioactivation pathway is feasible. To this end, the timing of mechanistic investigations is important; if carried out at an early stage of drug discovery, rationalization of the steps leading to P450 inactivation allows for medicinal chemistry to introduce structural modifications that may avoid this liability. Therefore, there is high value in experiments and workflows that can rapidly characterize P450 MBI mechanisms for new drug candidates. Here, we illustrate that the typical workflow (enzyme activity, UV-spectroscopy, heme quantitation, and protein mass spectrometry) can lead to ambiguous results, and further investigation revealed insights to explain the relationship between CYP3A4 alkylation, loss of enzyme catalytic function, and apparent heme loss.

The first line of detection for CYP3A4 MBI is measurement of enzyme activity. In this case, we observed that PIA and PM, compounds remarkably similar in structure and electrophilic reactivity, showed inactivation kinetics that were in stark contrast. PM inactivation of CYP3A4 was potent and rapid, whereas PIA had a negligible effect on enzyme activity. Pyrene is a known CYP3A4 substrate, and as such, the pyrenyl moiety increases the affinity of the tool molecules toward the enzyme active site. Also, because both PIA and PM possess highly reactive groups (soft electrophiles), the compounds were chosen to selectively alkylate the cysteine residues of the protein while avoiding cross-reacting with the active site heme group. Taken together, these observations provide evidence that alkylation of CYP3A4 does not necessitate loss of enzyme function but rather the loss of activity is compound-dependent.

To investigate if both PM and PIA alkylate the protein efficiently, we analyzed the reacted protein by intact mass spectrometry. After a 10minute incubation, both compounds completely adducted the protein (Fig. 3). In the case of PIA, a single molecule was covalently adducted to CYP3A4. Interestingly, PM alkylation afforded a heterogenous mixture of two adducted forms, the largest of which had a molecular weight corresponding to alkylation by a total of seven PM molecules, presumably covalently bonded to the seven cysteine residues in CYP3A4. The result was somewhat unexpected in that even Cysteine 442 was alkylated by PM; this residue is inaccessible as a nucleophile given that this amino acid is coordinated to the fifth axial position of the heme iron. Not only does this interaction anchor the heme group in the $\mathrm{P} 450$ active site but it also serves to modulate the electronic environment of the heme to facilitate the reduction potential of the group and consequently governs the overall catalytic function of the protein. As such, it was integral to our study to fully understand any effect PIA or PM have on CYP3A4 spectral properties and amount of intact heme.
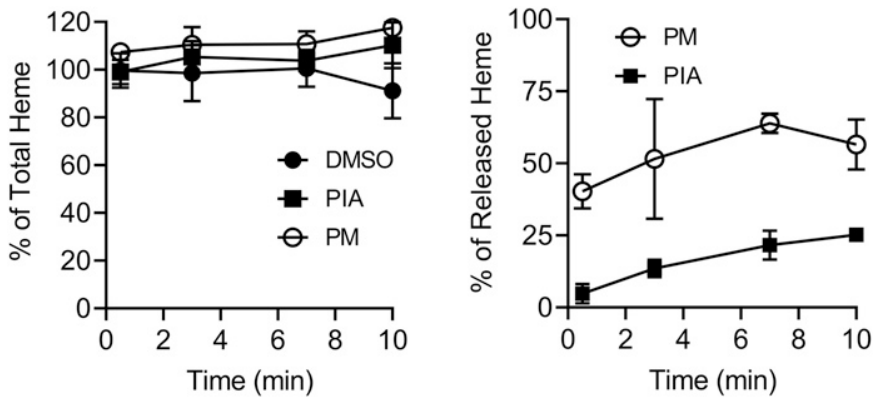

Fig. 6. Free heme release from the CYP3A4 after PM and PIA treatment. CYP3A4 Supersomes $(40 \mathrm{pmol} / \mathrm{ml}$, without b5) were incubated with vehicle $(0.5 \%$ DMSO), PM $(50 \mu \mathrm{M})$, and PIA $(50 \mu \mathrm{M})$ in separate incubations. The incubations were quenched, and the total and free heme were differentially extracted as described in Materials and Methods. Left panel shows the time course of total intact heme, whereas the right panel shows the percentage of heme released into solution over time. Points and error bars represent the mean of triplicate experiments \pm S.E.

Because of the unique features of the heme within the $\mathrm{P} 450$ active site, the use of UV spectrophotometric experiments is often a useful first step in dissecting the mechanistic basis of an observed P450 MBI. The hallmark of cytochrome P450 is the observed Soret shift from $\sim 418$ (for ferric, low-spin enzyme) to $\sim 450 \mathrm{~nm}$ (for the FeII-CO complex) upon binding of carbon monoxide to the sixth coordination position of the heme (Schenkman and Jansson, 2006). It has long been established that the observed P450-to-P420 spectral transition is a diagnostic indicator of changes in architectural integrity of P450 active site surrounding the heme; it reflects a separation of the iron-thiolate bond linking the prosthetic heme group to the P450 apoprotein (Lin et al., 2012; Sun et al., 2013). This can be achieved in vitro by multiple experimental conditions, including heat and detergents (Omura and Sato, 1964; Imai and Sato, 1967). Our spectroscopic data for PM is consistent with this unfolding mechanism, in which we observed a rapid decrease in P450 absorbance and a rapid increase in P420 (Fig. 5). In contrast, PIA treatment had a negligible effect on P450 absorbance. Similarly, we observed the liberation of the active site heme into the solution with PM but not with PIA (Fig. 6). In this light, as it is well-established that partial denaturation of a protein through small molecule adduction can precipitate a loss of the protein's function because of structural changes, the notion that the coordination and binding of the heme within the CYP3A4 active could be readily dissociated through a covalent modification and hence thermodynamic destabilization of the protein appears reasonable.

To further test the hypothesis that PM may lead to CYP3A4 protein unfolding, the effect of both PM and PIA treatment on CYP3A4 stability was examined using a DSF thermal shift assay. Unfortunately, pyrene compounds are fluorescent in nature, and the subsequent interference in signal precluded measuring melting temperature. However, using lightscattering measurements, we were able to confirm that by treating CYP3A4 with PM, the onset temperature of aggregation, $\mathrm{T}_{\mathrm{agg}}$, was

TABLE 1

Effect of PIA and PM pretreatment on recombinant CYP3A4 onset $\mathrm{T}_{\text {agg }}$ Values represent means of duplicate determinations.

\begin{tabular}{lc}
\hline Treatment & $\begin{array}{c}\text { Onset } \\
\mathrm{T}_{\text {agg }}\left({ }^{\circ} \mathrm{C}\right)\end{array}$ \\
\hline DMSO & 48.8 \\
Pyrene & 48.6 \\
Pyrene iodoacetamide & 48.3 \\
Pyrene maleimide & 36.0 \\
\hline
\end{tabular}


A

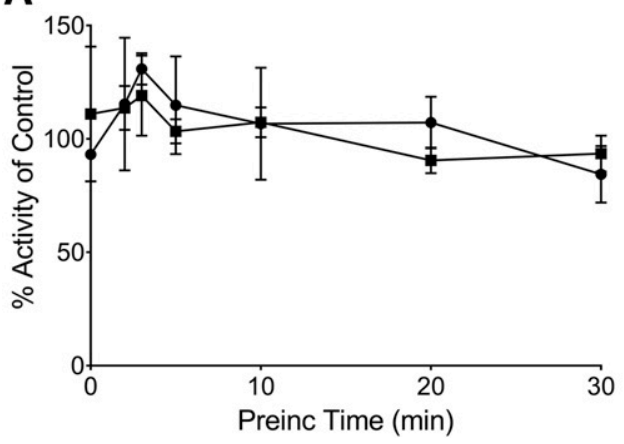

B

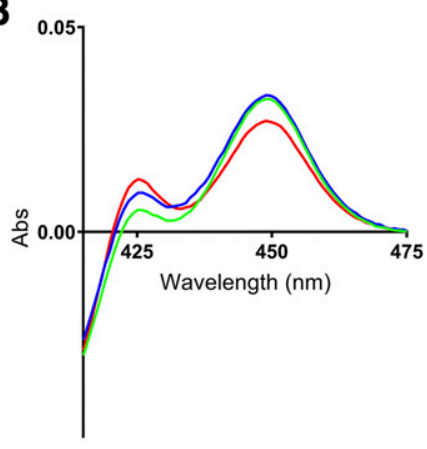

Fig. 7. Effect of PIA and PM pretreatment on recombinant $3 \mathrm{~A} 5$ activity and carbon monoxide binding spectra. (A) A solution containing CYP3A5 Supersomes in phosphate buffer was preincubated with PM or PIA $(50 \mu \mathrm{M})$ for various incubation times. After preincubation, mixture solutions were 20-fold diluted into a secondary incubation containing $10 \mu \mathrm{M}$ midazolam. Formation of 1-hydroxymidazolam was monitored, and the activity was calculated as a percentage of the product formation observed relative to vehicle-treated control at each time point. Transformed data for relative reaction rate of triplicate determinations \pm S.E. is shown. Circles and squares denote treatment by PM and PIA, respectively. (B) Incubations containing CYP3A5 Supersomes were treated with PM $(50 \mu \mathrm{M}$, red line), PIA $(50 \mu \mathrm{M}$, blue line), or DMSO (green line) for 5 minutes at $37^{\circ} \mathrm{C}$. CO binding was measured using UV-visible spectrometer difference spectra scanning from 400 to $500 \mathrm{~nm}$ as described in Materials and Methods. greater than $10^{\circ} \mathrm{C}$ lower relative to PIA or negative controls. The results of the DSF studies to monitor structural disruption studies also suggest that the integrity of the CYP3A4 protein structure was markedly lost upon adduction of the PM compared with PIA. Furthermore, these data establish that for some molecules, covalent adduction to the CYP3A4 active site precipitates moderate protein unfolding, which leads to exposure of hydrophobic regions of the protein that ultimately leads to protein aggregation, as observed with the pyrene maleimide adducted CYP3A4.

To gain further insight into the specific residues that may be implicated in the CYP3A4 protein destabilization process, we examined the possibility of time-dependent inactivation upon incubation with both PIA and PM with CYP3A5. This line of investigation using CYP3A5 appeared appropriate, as this protein possesses approximately $83 \%$ sequence similarity with CYP3A4 and typically shares a high degree of overlapping substrate specificity with CYP3A4 as well (Watanabe et al., 2017). Interestingly, neither compound precipitated CYP3A5 activity loss (Fig. 7). CYP3A4 has six cysteines that may react directly with an electrophilic small molecule (Cys58, Cys64, Cys98, Cys239, Cys377, and Cys468), whereas CYP3A5 has only four that are reactive (Cys64, Cys 98, Cys377, and Cys467) All cysteines other than Cys58 and Cys239 are conserved in CYP3A5, which indicates that one of these residues may be involved in initiating the CYP3A4 MBI cascade. Previously, it was found that the accessibility to Cysteine 58 by a small molecule was poor (Tsalkova et al., 2007; Sineva et al., 2013). In contrast, Cysteine 239 is freely accessible, residing along the large and malleable channel near the active site of CYP3A4. In fact, Cysteine 239 is an important residue linked to in vitro P450 MBI by marketed drugs and drug candidates such as raloxifene and AMG 487 (Baer et al., 2007; Henne et al., 2012; VandenBrink et al., 2012).

These data, when combined, support a hypothesis that the CYP3A4 Cysteine 239 may be involved in alkylation by PM, which may trigger a sequela of events that leads to protein destabilization, unfolding, and subsequent aggregation of the P450 enzyme. During this process, the active site heme environment is disrupted and, as a consequence, heme is liberated from the enzyme active site into solution, which accounts for the observed increase in heme after PM treatment. More generally, we have demonstrated: 1) alkylation of CYP3A4 does not necessarily precipitate loss of enzyme function, and 2) loss of $\mathrm{CO}$ binding can be a consequence of apoprotein adduction if the adduct leads to perturbations in the active site and local heme environment. The data highlight that subtle differences in compound structure and reactivity may have profound downstream effects on enzyme function and stability. Furthermore, this unfolding mechanism may have broader implications on hapten formation, which may contribute to adverse drug reactions associated with immunogenic response (Hess and Rieder, 1997; Pirmohamed et al., 1998).
It should be noted that the proposed hypothesis for inactivation could arise from other mechanisms. It is possible that PM simply has altered accessibility and reactivity to other residues in CYP3A4. However, this would suggest that the altered reactivity profile was unique to CYP3A4 relative to CYP3A5, which is conceivable given the structural differences between the isoforms. Previously, it was found that stoichiometrically controlled levels of PIA preferentially reacted with Cys239 (Baer et al., 2007). Similar experiments were undertaken with PM, and multiple cysteine adducts were detected with CYP3A4 (data not shown). However, there was no evidence of PM reactions with the other nucleophilic amino acids such as proline or lysine. This does not rule out their formation and may be due to the inability to observe such adducts because of instability or insufficient analytical sensitivity for detection.

In conclusion, small molecule adduction to CYP3A4: 1) can lead to loss of enzyme activity because of a structural change to the enzyme active site, prohibiting access of another substrate to the heme as observed with raloxifene and AMG 487;2) can abolish the CYP3A4 tertiary structure, causing it to unravel and aggregate as in the case of PM; or 3) as observed with PIA, is a relatively benign event. With respect to the latter, it should be noted that CYP3A4 is well-documented to adopt different conformations when bound to chemically distinct substrates. The limited use of a single-probe substrate in the current study does not preclude that the adduction by PIA does block the ligand entrance route for alternative CYP3A4 substrates. However, the finding of this study is consistent with a previous study in which PIA-treated CYP3A4 and untreated CYP3A4 produced similar amounts of the raloxifene metabolites (Baer et al., 2007). The work described in this manuscript reinforces the critical importance of describing the physical chemical mechanism that forms the basis of P450 MBI and highlights the need to fully understand the thermodynamic and colloidal stability of an adducted protein. The significance of thoroughly understanding the basis P450 MBI extends beyond a drug-metabolizing enzyme being nonfunctional, as in some cases unfolded and/or aggregated forms of a protein may potentially alter immunogenicity (Lopez Garcia et al., 1993; Kang et al., 2008; Metushi et al., 2014a,b).

\section{Acknowledgments}

We thank Jeffrey Jones and Carolyn Joswig-Jones for the gift of purified recombinant CYP3A4 protein

\section{Authorship Contributions}

Participated in research design: Barr, Wang, Min, H.J. Wienkers, B.M. Rock, D.A. Rock, L.C. Wienkers.

Conducted experiments: Barr, Wang, Min, H.J. Wienkers, B.M. Rock, D.A. Rock. 
Performed data analysis: Barr, Wang, Min, H.J. Wienkers, B.M. Rock, D.A.

Rock, L.C. Wienkers.

Wrote or contributed to the writing of the manuscript: Barr, Wang, Min, H.J. Wienkers, B.M. Rock, D.A. Rock, L.C. Wienkers.

\section{References}

Baer BR, Wienkers LC, and Rock DA (2007) Time-dependent inactivation of P450 3A4 by raloxifene: identification of Cys239 as the site of apoprotein alkylation. Chem Res Toxicol $\mathbf{2 0}$ 954-964.

Barnaba C, Yadav J, Nagar S, Korzekwa K, and Jones JP (2016) Mechanism-based inhibition of CYP3A4 by podophyllotoxin: aging of an intermediate is important for in vitro/in vivo correlations. Mol Pharm 13:2833-2843.

Bateman KP, Baker J, Wilke M, Lee J, Leriche T, Seto C, Day S, Chauret N, Ouellet M, and Nicoll-Griffith DA (2004) Detection of covalent adducts to cytochrome P450 3A4 using liquid chromatography mass spectrometry. Chem Res Toxicol 17:1356-1361.

Dabrowski MJ, Schrag ML, Wienkers LC, and Atkins WM (2002) Pyrene.pyrene complexes at the active site of cytochrome P450 3A4: evidence for a multiple substrate binding site. J Am Chem Soc 124:11866-11867.

de Montellano PRO (2018) 1-Aminobenzotriazole: a mechanism-based cytochrome P450 inhibitor and probe of cytochrome P450 biology. Med Chem (Los Angeles) 8:38-65.

Espinas NA, Kobayashi K, Takahashi S, Mochizuki N, and Masuda T (2012) Evaluation of unbound free heme in plant cells by differential acetone extraction. Plant Cell Physiol 53: $1344-1354$.

Foti RS, Rock DA, Pearson JT, Wahlstrom JL, and Wienkers LC (2011) Mechanism-based inactivation of cytochrome P450 3A4 by mibefradil through heme destruction. Drug Metab Dispos 39:1188-1195.

Henne KR, Tran TB, VandenBrink BM, Rock DA, Aidasani DK, Subramanian R, Mason AK, Stresser DM, Teffera Y, Wong SG, et al. (2012) Sequential metabolism of AMG 487, a novel CXCR3 antagonist, results in formation of quinone reactive metabolites that covalently modify CYP3A4 Cys239 and cause time-dependent inhibition of the enzyme. Drug Metab Dispos 40 1429-1440.

Hess DA and Rieder MJ (1997) The role of reactive drug metabolites in immune-mediated adverse drug reactions. Ann Pharmacother 31:1378-1387.

Imai Y and Sato R (1967) Conversion of P-450 to P-420 by neutral salts and some other reagents. Eur J Biochem 1:419-426.

Jushchyshyn MI, Hutzler JM, Schrag ML, and Wienkers LC (2005) Catalytic turnover of pyrene by CYP3A4: evidence that cytochrome b5 directly induces positive cooperativity. Arch Biochem Biophys 438:21-28.

Kang P, Liao M, Wester MR, Leeder JS, Pearce RE, and Correia MA (2008) CYP3A4-mediated carbamazepine (CBZ) metabolism: formation of a covalent CBZ-CYP3A4 adduct and alteration of the enzyme kinetic profile. Drug Metab Dispos 36:490-499.

Lin HL, Kenaan C, Zhang H, and Hollenberg PF (2012) Reaction of human cytochrome P450 3A4 with peroxynitrite: nitrotyrosine formation on the proximal side impairs its interaction with NADPH-cytochrome P450 reductase. Chem Res Toxicol 25:2642-2653.

Lin HL, Kent UM, and Hollenberg PF (2002) Mechanism-based inactivation of cytochrome P450 3A4 by 17 alpha-ethynylestradiol: evidence for heme destruction and covalent binding to protein. J Pharmacol Exp Ther 301:160-167.

Lin HL, Zhang H, and Hollenberg PF (2018) Formation of both heme and apoprotein adducts contributes to the mechanism-based inactivation of human CYP2J2 by $17 \alpha$-ethynylestradiol. Drug Metab Dispos 46:813-822.

Lopez Garcia MP, Dansette PM, Valadon P, Amar C, Beaune PH, Guengerich FP, and Mansuy D (1993) Human-liver cytochromes P-450 expressed in yeast as tools for reactive-metabolite formation studies. Oxidative activation of tienilic acid by cytochromes P-450 $2 \mathrm{C} 9$ and $2 \mathrm{C} 10$. Eur J Biochem 213:223-232

Metushi IG, Lee WM, and Uetrecht J (2014a) IgG3 is the dominant subtype of anti-isoniazid antibodies in patients with isoniazid-induced liver failure. Chem Res Toxicol 27:738-740.

Metushi IG, Sanders C, Lee WM, and Uetrecht J; Acute Liver Study Group (2014b) Detection of anti-isoniazid and anti-cytochrome P450 antibodies in patients with isoniazid-induced liver failure. Hepatology 59:1084-1093.

Murray M and Murray K (2003) Mechanism-based inhibition of CYP activities in rat liver by fluoxetine and structurally similar alkylamines. Xenobiotica 33:973-987.

Omura T and Sato R (1964) The carbon monoxide-binding pigment of liver microsomes. I. Evidence for its hemoprotein nature. $J$ Biol Chem 239:2370-2378.

Orr ST, Ripp SL, Ballard TE, Henderson JL, Scott DO, Obach RS, Sun H, and Kalgutkar AS (2012) Mechanism-based inactivation (MBI) of cytochrome P450 enzymes: structure-activity relationships and discovery strategies to mitigate drug-drug interaction risks. J Med Chem 55:4896-4933.

Pirmohamed M, Breckenridge AM, Kitteringham NR, and Park BK (1998) Adverse drug reactions. BMJ 316:1295-1298.

Rock BM, Hengel SM, Rock DA, Wienkers LC, and Kunze KL (2014) Characterization of ritonavir-mediated inactivation of cytochrome P450 3A4. Mol Pharmacol 86:665-674.

Schenkman JB and Jansson I (2006) Spectral analyses of cytochromes P450. Methods Mol Biol 320:11-18.

Sineva EV, Rumfeldt JA, Halpert JR, and Davydov DR (2013) A large-scale allosteric transition in cytochrome P450 3A4 revealed by luminescence resonance energy transfer (LRET). PLoS One 8:e83898.

Stillman LC and Gassman ML (1978) Protoheme extraction from plant tissue. Anal Biochem 91 $166-172$.

Sun Y, Zeng W, Benabbas A, Ye X, Denisov I, Sligar SG, Du J, Dawson JH, and Champion PM (2013) Investigations of heme ligation and ligand switching in cytochromes p450 and p420. Biochemistry 52:5941-5951.

Temel DB, Landsman P, and Brader ML (2016) Orthogonal methods for characterizing the unfolding of therapeutic monoclonal antibodies: differential scanning calorimetry, isothermal chemical denaturation, and intrinsic fluorescence with concomitant static light scattering. Methods Enzymol 567:359-389.

Tsalkova TN, Davydova NY, Halpert JR, and Davydov DR (2007) Mechanism of interactions of alpha-naphthoflavone with cytochrome P450 3A4 explored with an engineered enzyme bearing a fluorescent probe. Biochemistry 46:106-119.

VandenBrink BM, Davis JA, Pearson JT, Foti RS, Wienkers LC, and Rock DA (2012) Cytochrome p450 architecture and cysteine nucleophile placement impact raloxifene-mediated mechanismbased inactivation. Mol Pharmacol 82:835-842.

VandenBrink BM and Isoherranen N (2010) The role of metabolites in predicting drug-drug interactions: focus on irreversible cytochrome P450 inhibition. Curr Opin Drug Discov Devel 13:66-77.

Watanabe A, Takakusa H, Kimura T, Inoue SI, Kusuhara H, and Ando O (2017) Difference in mechanism-based inhibition of cytochrome P450 3A4 and 3A5 by a series of fluoroquinolone antibacterial agents. Drug Metab Dispos 45:336-341.

Zhou SF (2008) Drugs behave as substrates, inhibitors and inducers of human cytochrome P450 3A4. Curr Drug Metab 9:310-322.

Zhou SF, Xue CC, Yu XQ, Li C, and Wang G (2007) Clinically important drug interactions potentially involving mechanism-based inhibition of cytochrome P450 3A4 and the role of therapeutic drug monitoring. Ther Drug Monit 29:687-710.

Address correspondence to: Dan A. Rock, Amgen Research, Pharmacokinetics and Drug Metabolism, Amgen Inc., 1120 Veteran's Blvd., South San Francisco, CA 94080. E-mail: drock@amgen.com 